\title{
Technical scenarios for the decarbonization of road transport
}

Stephan Neugebauer

European Road Transport Research Advisory Council (ERTRAC)

This manuscript is not available according to publishing restriction.

Thank you for your understanding. 